\title{
Generalized Low Rank Approximations of Matrices
}

JIEPING YE*

jieping@cs.umn.edu

Department of Computer Science \& Engineering,University of Minnesota-Twin Cities, Minneapolis, MN 55455, USA

Editor: Peter Flach

Published online: 12 August 2005

Abstract. The problem of computing low rank approximations of matrices is considered. The novel aspect of our approach is that the low rank approximations are on a collection of matrices. We formulate this as an optimization problem, which aims to minimize the reconstruction (approximation) error. To the best of our knowledge, the optimization problem proposed in this paper does not admit a closed form solution. We thus derive an iterative algorithm, namely GLRAM, which stands for the Generalized Low Rank Approximations of Matrices. GLRAM reduces the reconstruction error sequentially, and the resulting approximation is thus improved during successive iterations. Experimental results show that the algorithm converges rapidly.

We have conducted extensive experiments on image data to evaluate the effectiveness of the proposed algorithm and compare the computed low rank approximations with those obtained from traditional Singular Value Decomposition (SVD) based methods. The comparison is based on the reconstruction error, misclassification error rate, and computation time. Results show that GLRAM is competitive with SVD for classification, while it has a much lower computation cost. However, GLRAM results in a larger reconstruction error than SVD. To further reduce the reconstruction error, we study the combination of GLRAM and SVD, namely GLRAM + SVD, where SVD is preceded by GLRAM. Results show that when using the same number of reduced dimensions, GLRAM + SVD achieves significant reduction of the reconstruction error as compared to GLRAM, while keeping the computation cost low.

Keywords: singular value decomposition, matrix approximation, reconstruction error, classification

\section{Introduction}

The problem of dimensionality reduction has recently received broad attention in areas such as machine learning, computer vision, and information retrieval (Berry, Dumais, \& O'Brie, 1995; Castelli, Thomasian, \& Li, 2003; Deerwester et al., 1990; Dhillon \& Modha, 2001; Kleinberg \& Tomkins, 1999; Srebro \& Jaakkola, 2003). The goal of dimensionality reduction is to obtain more compact representations of the data with limited loss of information. Traditional algorithms for dimensionality reduction are based on the so-called vector space model. Under this model, each datum is modeled as a vector and the collection of data is modeled as a single data matrix, where each column of the data matrix corresponds to a data point and each row corresponds to a feature dimension. The representation of data by vectors in Euclidean space allows one to compute the similarity between data points, based 
on the Euclidean distance or some other similarity metric. The similarity metrics on data points naturally lead to similarity-based indexing by representing queries as vectors and searching for their nearest neighbors (Aggarwal, 2001; Castelli, Thomasian, \& Li, 2003).

A well-known technique for dimensionality reduction is the low rank approximation by the Singular Value Decomposition (SVD), also called Latent Semantic Indexing (LSI) in information retrieval (Berry, Dumais, \& O'Brie, 1995). An appealing property of this low rank approximation is that it achieves the smallest reconstruction error among all approximations with the same rank. Details can be found in Section 2. Some theoretical justification of the empirical success of LSI can be found in Papadimitriou et al. (1998), where it is shown that LSI works in the context of a simple probabilistic "Corpus-generating" model. However, applications of this technique to high-dimensional data, such as images and videos, quickly run up against practical computational limits, mainly due to the expensive SVD computation in both time and space for large matrices (Golub \& Van Loan, 1996).

Several incremental algorithms have been proposed in the past (Brand, 2002; Gu \& Eisenstat, 1993; Kanth et al., 1998) to deal with the high space complexity of SVD, where the data points are inserted incrementally to update the SVD. To the best of our knowledge, such algorithms come with no guarantees on the quality of the approximation produced. Random sampling can be applied to speed up the SVD computation. More details can be found in Achlioptas and McSherry (2001), Drineas et al. (1999) and Frieze, Kannan, \& Vempala (1998).

\subsection{Contributions}

In this paper, we present a novel approach to alleviate the expensive SVD computation. The novelty lies in a new data representation model. Under this model, each datum is represented as a matrix, instead of a vector, and the collection of data is represented as a collection of matrices, instead of a single data matrix. We formulate the problem of low rank approximations as a new optimization problem, which approximates a collection of matrices with matrices of lower rank. To the best of our knowledge, there is no closed form solution for the new optimization problem. We thus derive an iterative algorithm, namely GLRAM. Detailed mathematical justification for this iterative procedure is given in Section 3.

Both GLRAM and SVD aim to minimize the reconstruction error. The essential difference is that GLRAM applies a bilinear transformation on the data. Such a bilinear transformation is particularly appropriate for data in matrix form, and often leads to lower computation cost in comparison to SVD. We apply GLRAM on image compression and retrieval, where each image is represented in its native matrix form. To evaluate the proposed algorithm, we have conducted extensive experiments on five well-known image datasets: PIX, ORL, AR, PIE, and USPS, where USPS consists of images of handwritten digits and the other four are face image datasets. GLRAM is compared with SVD, as well as 2DPCA, a recently proposed algorithm for dimension reduction. (Details on 2DPCA can be found in Section 4.)

Results show that when using the same number of reduced dimensions, GLRAM is competitive with SVD for classification, while it has a much lower computation cost. However, GLRAM results in a larger reconstruction error than SVD. The underlying 
reason may be that GLRAM is able to utilize the locality property (e.g., smoothness in an image) intrinsic in the data, which leads to good classification performance. In terms of compression ratio ${ }^{1}$, GLRAM outperforms SVD, especially when the number of data points is relatively small compared to the number of dimensions. For large and high-dimensional datasets, the lack of available space becomes a critical issue. In this case, compression ratio is an important factor in evaluating different dimensionality reduction algorithms.

To further reduce the reconstruction error of GLRAM, we study the combination of GLRAM and SVD, namely GLRAM + SVD, which applies SVD after the intermediate dimensionality reduction stage using GLRAM. The essence of this composite algorithm is a further dimensionality reduction stage by SVD following GLRAM. Since SVD is applied to a low-dimensional space transformed by GLRAM, the second stage by SVD can be implemented efficiently. We apply this algorithm to image datasets and compare it with GLRAM and SVD. Results show that when using the same number of reduced dimensions, GLRAM + SVD achieves a significant reduction of the reconstruction error as compared to GLRAM, while keeping the computation cost small. The reconstruction error of GLRAM + SVD is close to that of SVD, especially when the intermediate dimension in the GLRAM stage is large, while it has a smaller computation cost than SVD.

In summary, GLRAM can be applied as a pre-processing step for SVD. The preprocessing by GLRAM reduces significantly the computation cost of the SVD computation, while keeping the reconstruction error small (see Section 5.6).

\subsection{Organization of the paper}

The rest of this paper is organized as follows. We give a brief overview of low rank approximations of matrices in Section 2. The problem of generalized low rank approximations of matrices is studied in Section 3. Some related work is presented in Section 4. A performance study is provided in Section 5. Conclusions and directions for future work can be found in Section 6.

A preliminary version of this paper appears in the Proceedings of the Twenty-First International Conference on Machine Learning, Alberta, Canada, pp. 887-894, 2004. This submission is substantially extended and contains: (1) additional datasets in Section 5.1, such as RAND and USPS; (2) new experiments in Sections 5.2-5.4; and (3) inclusion of GLRAM + SVD in Section 5.6.

The major notations used throughout the rest of this paper are listed in Table 1.

\section{Low rank approximations of matrices}

Traditional methods in information retrieval and machine learning deal with data in vectorized representation. A collection of data is then stored in a single matrix $A \in \mathbb{R}^{N \times n}$, where each column of $A$ corresponds to a vector in the $N$-dimensional space. A major benefit of this vector space model is that the algebraic structure of the vector space can be exploited (Berry, Dumais, \& O’Brie, 1995).

For high-dimensional data, one would like to simplify the data, so that traditional machine learning and statistical techniques can be applied. However, crucial information intrinsic 
Table 1. Notations.

\begin{tabular}{ll}
\hline Notations & \multicolumn{1}{c}{ Descriptions } \\
\hline$A_{i}$ & The $i$-th data point in matrix form \\
$r$ & Number of rows in $A_{i}$ \\
$c$ & Number of columns in $A_{i}$ \\
$L$ & Transformation on the left side \\
$R$ & Transformation on the right side \\
$M_{i}$ & Reduced representation of $A_{i}$ \\
$\ell_{1}$ & Number of rows in $M_{i}$ \\
$\ell_{2}$ & Number of columns in $M_{i}$ \\
$d$ & Common value for $\ell_{1}$ and $\ell_{2}$ \\
$k$ & Number of reduced dimensions by SVD \\
$A$ & Data matrix of size $N$ by $n$ \\
$n$ & Number of training data points \\
$N$ & Dimension of training data $(N=r c)$ \\
\hline
\end{tabular}

in the data should not be removed under this simplification. A widely used method for this purpose is to approximate the single data matrix, $A$, with a matrix of lower rank. Mathematically, the optimal rank- $k$ approximation of a matrix $A$, under the Frobenius norm can be formulated as follows:

Find a matrix $B \in \mathbb{R}^{N \times n}$ with $\operatorname{rank}(B)=k$, such that

$$
B=\arg \min _{\operatorname{rank}(\mathrm{B})=\mathrm{k}}\|A-B\|_{F},
$$

where the Frobenius norm, $\|M\|_{F}$, of a matrix $M=\left(M_{i j}\right)$ is given by $\|M\|_{F}=\sqrt{\sum_{i, j} M_{i j}^{2}}$. The matrix $B$ can be readily obtained by computing the Singular Value Decomposition (SVD) of $A$, as stated in the following theorem (Golub \& Van Loan, 1996).

Theorem 2.1. Let the Singular Value Decomposition of $A \in \mathbb{R}^{N \times n}$ be $A=U D V^{T}$, where $U$ and $V$ are orthogonal, $D=\operatorname{diag}\left(\sigma_{1}, \ldots, \sigma_{r}, 0, \ldots, 0\right), \sigma_{1} \geq \cdots \geq \sigma_{r}>0$ and $r$ $=\operatorname{rank}(A)$. Then for $1 \leq k \leq r, \sum_{i=k+1}^{r} \sigma_{i}^{2}=\min \left\{\|A-B\|_{F}^{2} \mid \operatorname{rank}(B)=k\right\}$. The minimum is achieved with $B=$ best $_{k}(A)$, where best $t_{k}(A)=U_{k} \operatorname{diag}\left(\sigma_{1}, \ldots, \sigma_{k}\right) V_{k}^{T}$, and $U_{k}$ and $V_{k}$ are the matrices formed by the first $k$ columns of $U$ and $V$ respectively.

For any approximation $M$ of $A$, we call $\|A-M\|_{F}$ the reconstruction error of the approximation. By Theorem 2.1, $B=U_{k} \operatorname{diag}\left(\sigma_{1}, \ldots, \sigma_{k}\right) V_{k}^{T}$ has the smallest reconstruction error among all the rank- $k$ approximations of $A$.

Under this approximation, each column, $a_{i} \in \mathbb{R}^{N}$, of $A$ can be approximated as $a_{i} \approx U_{k} a_{i}^{L}$, for some $a_{i}{ }^{L} \in \mathbb{R}^{k}$. Since $U_{k}$ has orthonormal columns, $\left\|U_{k} a_{i}{ }^{L}-U_{k} a_{j}{ }^{L}\right\|=\left\|a_{i}{ }^{L}-a_{j}{ }^{L}\right\|$, i.e., the Euclidean distance between two vectors are preserved under the orthogonal projection. It follows that $\left\|a_{i}-a_{j}\right\| \approx\left\|U_{k} a_{i}{ }^{L}-U_{k} a_{j}{ }^{L}\right\|=\left\|a_{i}{ }^{L}-a_{j}{ }^{L}\right\|$. Hence the proximity of $a_{i}$ and $a_{j}$, 
in the original high-dimensional space, can be approximated by computing the proximity of their reduced representations $a_{i}{ }^{L}$ and $a_{j}{ }^{L}$. The speed-up on a single distance computation using the reduced representations is $N / k$. This forms the basis for Latent Semantic Indexing (Berry, Dumais, \& O’Brie, 1995; Deerwester et al., 1990), used widely in informational retrieval.

Another potential application of the above rank- $k$ approximation is for data compression. Since each $a_{i}$ is approximated by $U_{k} a_{i}{ }^{L}$, where $U_{k}$ is common for every $a_{i}$, we need to keep $U_{k}$ and $\left\{a_{i}{ }^{L}\right\}_{i=1}^{n}$ only for all the approximations. Since $U_{k} \in \mathbb{R}^{N \times k}$ and $a_{i}{ }^{L} \in \mathbb{R}^{k}$, for $i=$ $1, \ldots, n$, it requires $n k+N k=(n+N) k$ scalars to store the reduced representations. The storage saved, or compression ratio, using the rank- $k$ approximation is thus $n N /(n+N) k$, since the original data matrix $A$ is of size $N$ by $n$.

\section{Generalized low rank approximations of matrices}

In this section, we study the problem of generalized low rank approximations of matrices, which aims to approximate a collection of matrices with lower rank. A key difference between this generalized problem and the low rank approximation problem discussed in the last section, is the data representation model applied.

Recall that the vector space model is applied for the traditional low rank approximations. The vector space model leads to a simple and closed form solution for low rank approximations by computing the SVD of the data matrix. However, the SVD computation restricts its applicability to matrices of small size. Instead, we apply a different data representation model, under which each datum is represented as a matrix and the collection of data is represented as a collection of matrices.

\subsection{Problem formulation}

Let $A_{i} \in \mathbb{R}^{r \times c}$, for $i=1, \ldots, n$, be the $n$ data points in the training set, where $r$ and $c$ denote the number of rows and columns respectively for each $A_{i}$. We aim to compute two matrices $L \in \mathbb{R}^{r \times \ell_{1}}$ and $R \in \mathbb{R}^{c \times \ell_{2}}$ with orthonormal columns, and $n$ matrices $M_{i} \in$ $\mathbb{R}^{\ell_{1} \times \ell_{2}}$, for $i=1, \ldots, n$, such that $L M_{i} R^{T}$ approximates $A_{i}$, for all $i$. Here, $\ell_{1}$ and $\ell_{2}$ are two pre-specified parameters that are best set to the same value, based on the experimental results in Section 5. Mathematically, we can formulate this as the following minimization problem: Computing optimal $L, R$ and $\left\{M_{i}\right\}_{i=1}^{n}$, which solve

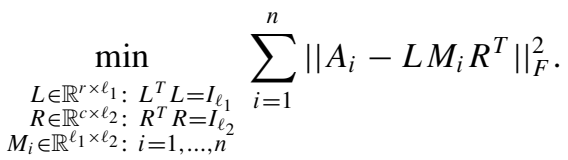

The matrices $L$ and $R$ in the above approximations act as the two-sided linear transformations on the data in matrix form. Recall that in the case of traditional low rank approximations, one-sided transformation is applied, which is $U_{k}$ in our previous discussions. Note that the $M_{i}$ 's are not required to be diagonal. 
The generalized low rank approximations above naturally lead to two basic applications.

- Data compression: The matrices $L, R$, and $\left\{M_{i}\right\}_{i=1}^{n}$ can be used to recover the original $n$ matrices $\left\{A_{i}\right\}_{i=1}^{n}$, assuming $L M_{i} R^{T}$ approximates $A_{i}$, for each $i$. It requires $r \ell_{1}+c \ell_{2}+n$ $\ell_{1} \ell_{2}$ scalars to store $L, R$, and $\left\{M_{i}\right\}_{i=1}^{n}$. Hence, the storage saved, or the compression ratio using the approximations is $n r c /\left(r \ell_{1}+c \ell_{2}+n \ell_{1} \ell_{2}\right)$.

- Distance computation: A common similarity metric on matrices is the Frobenius norm. The distance between $A_{i}$ and $A_{j}$ is $\left\|A_{i}-A_{j}\right\|_{F}$. Using the approximations, we have $\left\|A_{i}-A_{j}\right\|_{F} \approx\left\|L M_{i} R^{T}-L M_{j} R^{T}\right\|_{F}=\left\|M_{i}-M_{j}\right\|_{F}$, since both $L$ and $R$ have orthonormal columns. The computation cost of computing $\left\|A_{i}-A_{j}\right\|_{F}\left(\right.$ resp. $\left\|M_{i}-M_{j}\right\|_{F}$ ) is $O(r c)$ (resp. $O\left(\ell_{1} \ell_{2}\right)$ ). Hence, the speed-up on a single distance computation using the approximations is $r c /\left(\ell_{1} \ell_{2}\right)$.

Note that as $\ell_{1}$ and $\ell_{2}$ decrease, the speed-up on the distance computation and the compression ratio increase. However, small values of $\ell_{1}$ and $\ell_{2}$ may lead to loss of information intrinsic in the original data. We discuss this trade-off in Section 5.

The formulation in Eq. (1) is general, in the sense that $\ell_{1}$ and $\ell_{2}$ can be different, i.e., $M_{i}$ can have an arbitrary shape. We will study the effect of the shape of $M_{i}$ on the performance of the approximations in Section 5.2.

\subsection{The main algorithm}

In this section, we show how to solve the minimization problem in Eq. (1). The following theorem shows that the $M_{i}$ 's are determined by the transformation matrices $L$ and $R$, which significantly simplifies the minimization problem in Eq. (1).

Theorem 3.1. Let $L, R$ and $\left\{M_{i}\right\}_{i=1}^{n}$ be the optimal solution to the minimization problem in Eq. (1). Then $M_{i}=L^{T} A_{i} R$, for every $i$.

Proof: By the property of the trace of matrices,

$$
\begin{aligned}
\sum_{i=1}^{n}\left\|A_{i}-L M_{i} R^{T}\right\|_{F}^{2}= & \sum_{i=1}^{n} \operatorname{trace}\left(\left(A_{i}-L M_{i} R^{T}\right)\left(A_{i}-L M_{i} R^{T}\right)^{T}\right) \\
= & \sum_{i=1}^{n} \operatorname{trace}\left(A_{i} A_{i}^{T}\right)+\sum_{i=1}^{n} \operatorname{trace}\left(M_{i} M_{i}^{T}\right) \\
& -2 \sum_{i=1}^{n} \operatorname{trace}\left(L M_{i} R^{T} A_{i}^{T}\right),
\end{aligned}
$$

where the second term $\sum_{i=1}^{n} \operatorname{trace}\left(M_{i} M_{i}^{T}\right)$ results from the fact that both $L$ and $R$ have orthonormal columns, and $\operatorname{trace}(A B)=\operatorname{trace}(B A)$, for any two matrices. 
Since the first term on the right hand side of Eq. (2) is a constant, the minimization in Eq. (1) is equivalent to minimizing

$$
\sum_{i=1}^{n} \operatorname{trace}\left(M_{i} M_{i}^{T}\right)-2 \sum_{i=1}^{n} \operatorname{trace}\left(L M_{i} R^{T} A_{i}^{T}\right) .
$$

It is easy to check that the minimum of (3) is achieved, only if $M_{i}=L^{T} A_{i} R$, for every $i$. This completes the proof of the theorem.

Theorem 3.1 implies that $M_{i}$ is uniquely determined by $L$ and $R$ with $M_{i}=L^{T} A_{i} R$, for all $i$. Hence the key step for the minimization in Eq. (1) is the computation of the common transformations $L$ and $R$. A key property of the optimal transformations $L$ and $R$ is stated in the following theorem:

Theorem 3.2. Let $L, R$ and $\left\{M_{i}\right\}_{i=1}^{n}$ be the optimal solution to the minimization problem in Eq. (1). Then L and $R$ solve the following optimization problem:

$$
\max _{\substack{L \in \mathbb{R}^{r \times \ell_{1}}: L^{T} L=I_{\ell_{1}} \\ R \in \mathbb{R}^{c \times \ell_{2}}: R^{T} R=I_{\ell_{2}}}} \sum_{i=1}^{n}\left\|L^{T} A_{i} R\right\|_{F}^{2}
$$

Proof: From Theorem 3.1, $M_{i}=L^{T} A_{i} R$, for every $i$. Substituting this into $\sum_{i=1}^{n} \| A_{i}$ $L M_{i} R^{T} \|_{F}^{2}$, we obtain

$$
\sum_{i=1}^{n}\left\|A_{i}-L M_{i} R^{T}\right\|_{F}^{2}=\sum_{i=1}^{n}\left\|A_{i}\right\|_{F}^{2}-\sum_{i=1}^{n}\left\|L^{T} A_{i} R\right\|_{F}^{2} .
$$

Hence the minimization in Eq. (1) is equivalent to the maximization of

$$
\sum_{i=1}^{n}\left\|L^{T} A_{i} R\right\|_{F}^{2}
$$

which completes the proof of the theorem.

To the best of our knowledge, there is no closed form solution for the maximization problem in Eq. (4). A key observation, which leads to an iterative algorithm for the computation of $L$ and $R$, is stated in the following theorem:

Theorem 3.3. Let $L, R$ and $\left\{M_{i}\right\}_{i=1}^{n}$ be the optimal solution to the minimization problem in Eq. (1). Then

(1) For a given $R, L$ consists of the $\ell_{1}$ eigenvectors of the matrix

$$
M_{L}=\sum_{i=1}^{n} A_{i} R R^{T} A_{i}^{T}
$$


corresponding to the largest $\ell_{1}$ eigenvalues.

(2) For a given $L, R$ consists of the $\ell_{2}$ eigenvectors of the matrix

$$
M_{R}=\sum_{i=1}^{n} A_{i}^{T} L L^{T} A_{i}
$$

corresponding to the largest $\ell_{2}$ eigenvalues.

Proof: $\quad$ By Theorem 3.2, $L$ and $R$ maximize

$$
\sum_{i=1}^{n}\left\|L^{T} A_{i} R\right\|_{F}^{2}
$$

which can be rewritten as

$$
\begin{aligned}
\sum_{i=1}^{n} \operatorname{trace}\left(L^{T} A_{i} R R^{T} A_{i}^{T} L\right) & =\operatorname{trace}\left(L^{T} \sum_{i=1}^{n}\left(A_{i} R R^{T} A_{i}^{T}\right) L\right) \\
& =\operatorname{trace}\left(L^{T} M_{L} L\right),
\end{aligned}
$$

where $M_{L}=\sum_{i=1}^{n} A_{i} R R^{T} A_{i}^{T}$. Hence, for a given $R$, the maximum of

$$
\sum_{i=1}^{n}\left\|L^{T} A_{i} R\right\|_{F}^{2}=\operatorname{trace}\left(L^{T} M_{L} L\right)
$$

is achieved, only if $L \in \mathbb{R}^{r \times \ell_{1}}$ consists of the $\ell_{1}$ eigenvectors of the matrix $M_{L}$ corresponding to the largest $\ell_{1}$ eigenvalues. The maximization of trace $\left(L^{T} M_{L} L\right)$ can be considered as a special case of the more general optimization problem in Edelman, Arias and Smith (1998).

Similarly, by the property of the trace of matrices,

$$
\sum_{i=1}^{n}\left\|L^{T} A_{i} R\right\|_{F}^{2}
$$

can also be rewritten as

$$
\begin{aligned}
\sum_{i=1}^{n} \operatorname{trace}\left(R^{T} A_{i}^{T} L L^{T} A_{i} R\right) & =\operatorname{trace}\left(R^{T} \sum_{i=1}^{n}\left(A_{i}^{T} L L^{T} A_{i}\right) R\right) \\
& =\operatorname{trace}\left(R^{T} M_{R} R\right),
\end{aligned}
$$

where $M_{R}=\sum_{i=1}^{n} A_{i}^{T} L L^{T} A_{i}$. Hence, for a given $L$, the maximum of

$$
\sum_{i=1}^{n}\left\|L^{T} A_{i} R\right\|_{F}^{2}=\operatorname{trace}\left(R^{T} M_{R} R\right)
$$


is achieved, only if $R \in \mathbb{R}^{c \times \ell_{2}}$ consists of the $\ell_{2}$ eigenvectors of the matrix $M_{R}$ corresponding to the largest $\ell_{2}$ eigenvalues. This completes the proof of the theorem.

Theorem 3.3 results in an iterative procedure for computing $L$ and $R$ as follows: for a given $L$, we can compute $R$ by computing the eigenvectors of the matrix $M_{R}$; with the computed $R$, we can then update $L$ by computing the eigenvectors of the matrix $M_{L}$. The procedure can be repeated until convergence. The pseudo-code of the above iterative procedure is given in Algorithm GLRAM below.

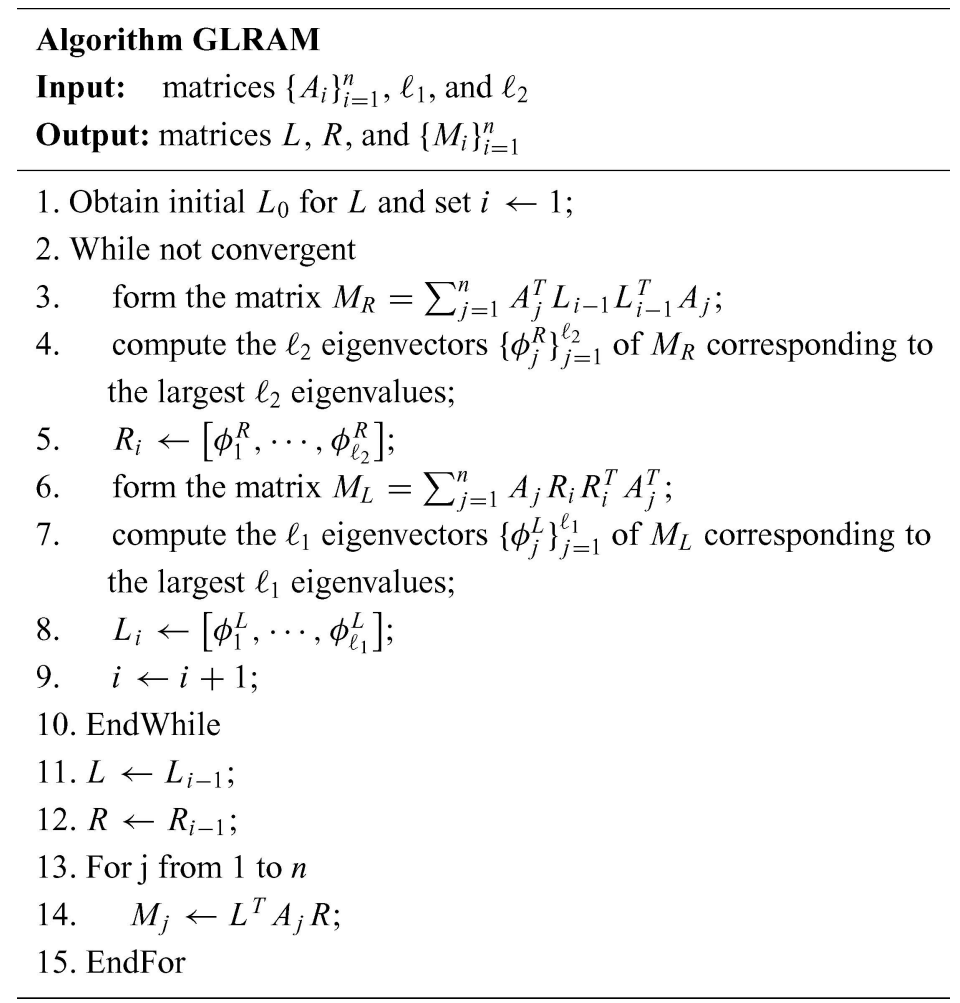

Theoretically, the solution to GLRAM is only locally optimal. The solution depends on the choice of the initial $L_{0}$ for $L$. We did extensive experiments (see Section 5.3) using different choices of the initial $L_{0}$ and found out that, for image datasets, GLRAM always converges to the same solution, regardless of the choice of the initial $L_{0}$.

Theorem 3.3 implies that the matrix updates in Lines 5 and 8 of GLRAM do not decrease the value of $\sum_{i=1}^{n}\left\|L^{T} A_{i} R\right\|_{F}^{2}$, since the computed $R$ and $L$ are locally optimal. Hence by Theorem 3.2, the value of $\sum_{i=1}^{n}\left\|A_{i}-L M_{i} R^{T}\right\|_{F}^{2}$, or

$$
\mathrm{RMSRE} \equiv \sqrt{\frac{1}{n} \sum_{i=1}^{n}\left\|A_{i}-L M_{i} R^{T}\right\|_{F}^{2}}
$$


does not increase. Here RMSRE stands for the Root Mean Square Reconstruction Error. The convergence of GLRAM follows, since RMSRE is bounded from below by 0 , as stated in the following Theorem:

Theorem 3.4. The GLRAM Algorithm monotonically non-increases the RMSRE value as defined in Eq. (8), hence it converges in the limit.

Thus we use the relative reduction of the RMSRE value to check the convergence. Specifically, let RMSRE( $i$ ) be the RMSRE value at the $i$-th iteration of the GLRAM algorithm, then the convergence of the algorithm is determined by checking whether the following inequality holds:

$$
\frac{\operatorname{RMSRE}(i-1)-\operatorname{RMSRE}(i)}{\operatorname{RMSRE}(i-1)}<\eta,
$$

for some small threshold $\eta>0$. In our experiments, we choose $\eta=10^{-6}$. Results in Section 5 show that the algorithm converges within two to three iterations.

Note that the transformation matrices $L$ and $R$ in GLRAM may not converge, even when the RMSRE value converges. To see why this is the case, consider two pairs of solutions $(L, R)$ and $(L P, R Q)$, for some orthogonal matrices $P \in \mathbb{R}^{\ell_{1} \times \ell_{1}}$ and $Q \in \mathbb{R}^{\ell_{2} \times \ell_{2}}$. Since

$$
\mathrm{RMSRE} \equiv \sqrt{\frac{1}{n} \sum_{i=1}^{n}\left\|A_{i}-L M_{i} R^{T}\right\|_{F}^{2}}=\sqrt{\frac{1}{n} \sum_{i=1}^{n}\left\|A_{i}-L L^{T} A_{i} R R^{T}\right\|_{F}^{2}},
$$

it is easy to verify that both $(L, R)$ and $(L P, R Q)$ result in the same RMSRE value. Thus, the solution to GLRAM is invariant under arbitrary orthogonal transformations. Two transformations $L$ and $\hat{L}$ can be compared by computing the largest principal angle ((Bjork \& Golub, 1973; Golub \& Van Loan, 1996) between the column spaces of $L$ and $\hat{L}$. If the angle is zero, $L$ is essentially equivalent to $\hat{L}$ up to an orthogonal transformation.

\subsection{Time and space complexities}

The most expensive steps in GLRAM are the formation of the matrices $M_{R}$ and $M_{L}$ in Lines 3 and 6, and the formation of $M_{j}$ in Lines 13-15.

It takes $O\left(\ell_{1} c(r+c) n\right)$ time for computing $M_{R}$ and $O\left(\ell_{2} r(r+c) n\right)$ time for computing $M_{L}$. The computation time of $M_{j}=\left(L^{T}\left(A_{j} R\right)\right)$ using the given order is $O\left(r c \ell_{2}+r \ell_{2} \ell_{1}\right)=$ $O\left(r \ell_{2}\left(c+\ell_{1}\right)\right)$. Assume the number of iterations in the while loop (from Line 2 to Line $10)$ is $I$. The total time complexity of GLRAM is $O\left(I(r+c)^{2} \max \left(\ell_{1}, \ell_{2}\right) n\right)$.

It is easy to verify that the space complexity of GLRAM is $O(r c)=O(N)$. The key to the low space complexity is that the formation of the matrices $M_{R}$ and $M_{L}$ can be proceeded by reading the matrices $\left\{A_{i}\right\}_{i=1}^{n}$ incrementally.

Note that GLRAM involves eigenvalue problems of size $r^{2}$ or $c^{2}$, as compared to size $r c n(=N n)$ in SVD. This is the key reason why GLRAM has much lower costs in time and space than SVD. 


\section{Related work}

Wavelet transform is a commonly used scheme for image compression (Averbuch, Lazar, \& Israeli, 1996). Similar to the GLRAM algorithm in this paper, wavelets can be applied to images in matrix representation. A subtle but important difference between wavelet compression and GLRAM compression is that the former mainly aims to compress and reconstruct a single image with small cost of basis representations, which is extremely important for image transmission in computer networks, whereas GLRAM compression aims to compress a set of images by making use of the correlation information between images.

A collection of images can also be considered as a 3rd-order tensor, or three-dimensional array. Decomposition of higher-order tensors has been studied in Kolda (2001), Shashua and Levin (2001), Vasilescu and Terzopoulos (2002), and Zhang and Golub (2001). Our approach differs in that we keep explicit the $2 \mathrm{D}$ nature of images.

The work that is most closely related to the current one is the two-dimensional Principal Component Analysis (2DPCA) algorithm recently proposed in Yang et al. (2004). Like GLRAM, 2DPCA works with data in matrix form. The key difference is that 2DPCA applies linear transformation on the right side of the data, while GLRAM applies two-sided linear transformation. 2DPCA can be formulated as a trace optimization problem, from which a closed form solution is obtained. However, a disadvantage of 2DPCA, as also mentioned in Yang et al. (2004), is that the number of reduced dimensions of 2DPCA can be quite large. More details are given below.

2DPCA computes a linear transformation $X \in \mathbb{R}^{c \times \ell}$ with $\ell<c$, such that each image $A_{i}$ $\in \mathbb{R}^{r \times c}$ is transformed (projected) to $Y_{i}=A_{i} X \in \mathbb{R}^{r \times \ell}$. The variance of the $n$ projections $\left\{Y_{i}\right\}_{i=1}^{n}$ can be computed as

$$
\frac{1}{n-1} \sum_{i=1}^{n}\left\|Y_{i}-\bar{Y}\right\|_{F}^{2}=\frac{1}{n-1} \sum_{i=1}^{n} X^{T}\left(A_{i}-\bar{A}\right)^{T}\left(A_{i}-\bar{A}\right) X
$$

where $\bar{Y}=\frac{1}{n} \sum_{i=1}^{n} Y_{i}=\bar{A} X$ is the mean and $\bar{A}=\frac{1}{n} \sum_{i=1}^{n} A_{i}$.

The optimal transformation $X$ in 2DPCA is computed such that the variance of the $n$ data points in the transformed space is maximized. Specifically, the optimal transformation $X$ can be computed by solving the following maximization problem:

$$
X=\arg \max _{X^{T} X=I_{\ell}}\left(\frac{1}{n-1} \sum_{i=1}^{n} X^{T}\left(A_{i}-\bar{A}\right)^{T}\left(A_{i}-\bar{A}\right) X\right)
$$

The optimal $X$ can be obtained by computing the $\ell$ eigenvectors of the matrix $\frac{1}{n-1} \sum_{i=1}^{n}\left(A_{i}-\right.$ $\bar{A})^{T}\left(A_{i}-\bar{A}\right)$ corresponding to the largest $\ell$ eigenvalues.

It requires $c \ell+n r \ell$ scalars to store $X \in \mathbb{R}^{c \times \ell}$ and $\left\{Y_{i}\right\}_{i=1}^{n} \in \mathbb{R}^{r \times \ell}$. Hence, the compression ratio by $2 \mathrm{DPCA}$ is $n r c /(c \ell+n r \ell) \approx c / \ell$.

Table 2 lists the time and space complexities of SVD, 2DPCA, and GLRAM. It is clear that GLRAM and 2DPCA have much smaller costs in time and space than SVD. 
Table 2. Comparison of SVD, 2DPCA, and GLRAM: $n$ is the number of data points in the training dataset and $N=r \times c$ is the dimension of the data.

\begin{tabular}{lll}
\hline Methods & \multicolumn{1}{c}{ Time } & Space \\
\hline SVD & $O(n N \min (n, N))$ & $O(n N)$ \\
2DPCA & $O\left(n c^{2} r\right)$ & $O(N)$ \\
GLRAM & $O\left(I(r+c)^{2} \max \left(\ell_{1}, \ell_{2}\right) n\right)$ & $O(N)$ \\
\hline
\end{tabular}

\section{Experimental evaluations}

In this section, we experimentally evaluate the GLRAM algorithm. All of our experiments are performed on a P4 $2.785 \mathrm{GHz}$ Linux machine with 1GB memory. A MATLAB version of the GLRAM algorithm can be accessed at http://wwwusers.cs. umn. edu/ jieping/GLRAM/\}.

We present in Section 5.1 one synthetic dataset and five real-world image datasets used for our evaluation. The effect of the ratio of $\ell_{1}$ to $\ell_{2}$ on reconstruction error is discussed in Section 5.2. Results show that, for the datasets considered in the paper, choosing $\ell_{1} / \ell_{2} \approx 1$ achieves good performance. We thus set both $\ell_{1}$ and $\ell_{2}$ equal to a common value $d$ in the following experiments. The sensitivity of GLRAM to the choice of the initial $L_{0}$ for $L$ is studied in Section 5.3. In Sections 5.4-5.5, a detailed comparative study between the proposed algorithm and SVD is provided, where the comparison is made on the reconstruction error (measured by RMSRE), classification, and quality of compressed images. The results with 2DPCA (Yang et al., 2004) are also included. The effectiveness of SVD critically depends on the reduced dimension $k$. For all the experiments, $k$ is chosen so that both SVD and GLRAM have the same number of reduced dimensions. Finally, we study the GLRAM + SVD algorithm in Section 5.6.

For all the experiments, we use the K-Nearest-Neighbors (K-NN) method with $K=1$ based on the Euclidean distance for classification (Duda, Hart, \& Stork, 2000; Fukunaga, 1990). We use 10-fold cross-validation for estimating the misclassification error rate. In 10-fold cross-validation, we divide the data into ten subsets of approximately equal size. Then we do the training and testing ten times, each time leaving out one of the subsets for training, and using only the omitted subset for testing. The misclassification error rate reported is the average from the ten runs.

\subsection{Datasets}

We use the following six datasets (one synthetic dataset and five real-world image datasets) in our experiments:

- RAND is a synthetic dataset, consisting of 500 data points of size $100 \times 100$. All the entries are randomly generated between 0 and 255 (the same range as the four face image datasets).

- PIX ${ }^{2}$ contains 300 face images of 30 persons. The size of PIX images is $512 \times 512$. We subsample the images down to a size of $100 \times 100=10000$. 
- $\mathrm{ORL}^{3}$ is a well-known dataset for face recognition (Samaria \& Harter, 1994). It contains the face images of 40 persons, for a total of 400 images. The image size is $92 \times 112$. The face images are perfectly centred. The major challenge posed by this dataset is the variation of the face pose. We use the whole image as an instance (i.e., the dimension of an instance is $92 \times 112=10304)$.

- $\mathrm{AR}^{4}$ is a large face image dataset (Martinez \& Benavente, 1998). The instance of each face may contain large areas of occlusion, due to the presence of sun glasses and scarves. The existence of occlusion dramatically increases the within-class variances of AR face image data. We use a subset of AR. This subset contains 1638 face images of 126 persons. Its image size is $768 \times 576$. We first crop the image from row 100 to 500 , and column 200 to 550, and then subsample the cropped images down to a size of $101 \times 88=8888$.

- PIE 5 is a subset of the CMU-PIE face image dataset (Sim et al., 2004). PIE contains 6615 face instances of 63 persons. More specifically, each person has $21 \times 5=105$ instances taken under 21 different lighting conditions and 5 different poses. The image size of PIE is $640 \times 480$. We pre-process each image using a similar technique as above. The final dimension of each instance is $32 \times 24=768$.

- USPS 6 is an image dataset consisting of 9298 handwritten digits of " 0 " through " 9 ". We use a subset of USPS. This subset contains 300 images for each digit, for a total of 3000 images. The image size is $16 \times 16=256$.

The statistics of all datasets are summarized in Table 3.

\subsection{Effect of the ratio of $\ell_{1}$ to $\ell_{2}$ on reconstruction error}

In this experiment, we study the effect of the ratio of $\ell_{1}$ to $\ell_{2}$ on reconstruction error, where $\ell_{1}$ and $\ell_{2}$ are the row and column dimensions of the reduced representation $M_{i}$ in GLRAM. To this end, we run GLRAM with different combinations of $\ell_{1}$ and $\ell_{2}$ with a constant product $\ell_{1} \cdot \ell_{2}=400$. The results on PIX, ORL, and AR are shown in Table 4. It is clear from the table that the RMSRE value is small, when $\ell_{1} / \ell_{2} \approx 1$, and the minimum is achieved when $\ell_{1} / \ell_{2}=1$ in all cases.

To examine whether this is related to the fact that for images, the number of rows $(r)$ and the number of columns $(c)$ are comparable, we subsample the images in PIX down to a size of $50 \times 100=5000$. The result on this dataset is included in Table 4. Interestingly, we observe the same trend in this dataset. That is, the RMSRE value is small, when $\ell_{1} / \ell_{2} \approx$

Table 3. Statistics of our test datasets.

\begin{tabular}{lccc}
\hline Dataset & Size $(n)$ & Dimension $(r \times c)$ & Number of classes \\
\hline RAND & 500 & $100 \times 100=10000$ & - \\
PIX & 300 & $100 \times 100=10000$ & 30 \\
ORL & 400 & $92 \times 112=10304$ & 40 \\
AR & 1638 & $101 \times 88=8888$ & 126 \\
PIE & 6615 & $32 \times 24=768$ & 63 \\
USPS & 3000 & $16 \times 16=256$ & 10 \\
\hline
\end{tabular}


1. We have conducted similar experiments on other datasets and observed the same trend. This may be related to the effect of balancing between the left and right transformations involved in GLRAM.

Finally, we examine the effect of the ratio using the synthetic dataset. The result on RAND is included in the last column of Table 4 . We observe the same trend as other datasets. That is, the RMSRE value is small, when $\ell_{1} / \ell_{2} \approx 1$.

The above experiment on both the synthetic and real-world datasets suggests that choosing $\ell_{1} / \ell_{2} \approx 1$ may be a good strategy in practice. In all the following experiments, we set both $\ell_{1}$ and $\ell_{2}$ equal to a common value $d$.

\subsection{Sensitivity of GLRAM to the choice of the initial $L_{0}$}

In this experiment, we examine the sensitivity of GLRAM to the choice of the initial $L_{0}$ for $L$ (see Line 1 of the GLRAM algorithm). To this end, we run GLRAM with 10 different initial $L_{0}$ 's. The first one is $L_{0}=\left(I_{d}, 0\right)^{T}$, while the next nine being randomly generated.

First, we study the sensitivity of GLRAM using the image datasets. The result on ORL is shown in figure 1 (left), where the horizontal axis is the number of iterations and the vertical axis is the RMSRE value (on a log scale). $d$ is set to be 10 .

We can observe from the figure that GLRAM converges rapidly for all ten initial choices of $L$. It converges within two to three iterations with the specified threshold $\left(\eta=10^{-6}\right)$. For all ten different initial $L_{0}$ 's, GLRAM converges to the same RMSRE value. To check whether GLRAM converges to the same solution, we compare the resulting left transformations $L$ from the ten different runs. Two transformations can be compared by computing the largest principal angle between the column spaces of these two transformations, as discussed in Section 3. The angle between the left transformation resulting from the first run and the ones from other nine runs are computed (results omitted). For all cases, the angles are around $10^{-10}$ to $10^{-7}$. This implies that GLRAM essentially converges to the same solution (subject to an orthogonal transformation) for the ten different runs. We observe the same

Table 4. Effect of the ratio of $\ell_{1}$ to $\ell_{2}$ on reconstruction error: Row shown in bold has minimum RMSRE (where $\ell_{1}=\ell_{2}$ ).

\begin{tabular}{rrrrrrr}
\hline \multicolumn{2}{c}{ Parameters } & & \multicolumn{5}{c}{ Datasets } \\
\cline { 2 - 6 }$\ell_{1}$ & $\ell_{2}$ & PIX & ORL & AR & PIX $(50 \times 100)$ & RAND \\
\hline 5 & 80 & 569.06 & 2128.8 & 3605.4 & 384.67 & 7189.9 \\
8 & 50 & 441.72 & 1737.2 & 2822.0 & 290.97 & 7177.5 \\
10 & 40 & 387.47 & 1580.1 & 2457.4 & 250.55 & 7174.1 \\
16 & 25 & 294.90 & 1376.9 & 1978.3 & 180.88 & 7170.9 \\
$\mathbf{2 0}$ & $\mathbf{2 0}$ & $\mathbf{2 7 8 . 0 1}$ & $\mathbf{1 3 6 7 . 3}$ & $\mathbf{1 9 0 2 . 8}$ & $\mathbf{1 6 9 . 2 8}$ & $\mathbf{7 1 7 0 . 6}$ \\
25 & 16 & 279.81 & 1423.9 & 1965.4 & 172.43 & 7171.1 \\
40 & 10 & 349.12 & 1697.6 & 2379.1 & 226.90 & 7174.2 \\
50 & 8 & 406.06 & 1864.6 & 2629.4 & 269.79 & 7177.2 \\
80 & 5 & 529.26 & 2366.1 & 3426.3 & - & 7190.0 \\
\hline
\end{tabular}


trend in other four image datasets (PIX, AR, PIE, and USPS) as well as different values of $d$ and the results are omitted.

Next, we examine the sensitivity of GLRAM using RAND, the synthetic dataset. The result is shown in figure 1 (right). It is clear from the figure that GLRAM converges much slower on RAND than on image datasets. We run GLRAM with the threshold $\eta=10^{-6}$, and it does not converge until 78 iterations. Furthermore, GLRAM does not converge to the same solution (measured by the angle between two subspaces). Further experiments also show that the final RMSRE value may be different for different initial $L_{0}$ 's, even though the difference always seems small. This is likely due to the fact that there are some similarities among the images in the same image datasets, while the data in RAND is randomly generated.

The experiment above implies that for datasets with some hidden structures, such as faces and handwritten digits, GLRAM may converge to the global solution, regardless of the choice of the initial $L_{0}$. However, it is not true in general, as shown in the RAND dataset.

\subsection{Comparison of reconstruction error and classification}

In this experiment, we evaluate the effectiveness of the proposed algorithm in terms of the reconstruction error measured by RMSRE and classification measured by misclassification error rate, and compare it with 2DPCA and SVD. For SVD, the reduced dimension $(k)$ is chosen so that both SVD and GLRAM have the same number of reduced dimensions, that is, $k=d^{2}$, where $d$ is the common value for both $\ell_{1}$ and $\ell_{2}$.

Figures 2-6 show the results on the five image datasets: PIX, ORL, AR, PIE, and USPS respectively. The horizontal axis denotes the value of $d$, and the vertical axis denotes the RMSRE value (left graph) and misclassification rate (right graph). Figure 7 shows the compression ratios of all algorithms on AR (left graph) and PIE (right graph), two representatives of all image datasets in Table 3.

The main observations include:


Figure 1. Sensitivity of GLRAM to the choice of the initial $L_{0}$ on ORL (left) and RAND (right). The ten curves correspond to the ten runs with different initial $L_{0}$ 's. The horizontal axis is the number of iterations and the vertical axis is the RMSRE value (on a log scale). 
- As $d$ increases, the reconstruction error by GLRAM decreases monotonically for all cases, while the misclassification rate decreases monotonically in most cases. The same trend can be observed from other algorithms. Thus choosing a large $d$ in general improves the performance of GLRAM in reconstruction and classification. However, the computation cost of GLRAM also increases as $d$ increases, as shown in Table 2 (Note that $d=\ell_{1}$ $=\ell_{2}$ ). There is a tradeoff between the performance and the computation cost, when choosing the best $d$ in GLRAM.

- SVD has the smallest RMSRE value in all cases, while 2DPCA has the largest RMSRE value in most cases. The large reconstruction error of 2DPCA is due to its poor compression performance, when using only one-sided transformation, as compared to two-sided transformation in GLRAM.

- For datasets with a relatively large number of dimensions compared to the number of data points, such as the AR datasets, the compression ratio of SVD is much smaller than others as shown in figure 7 (left graph). As the number of data points gets as large as in the PIE dataset, the compression ratio of SVD becomes close to GLRAM, as shown in figure 7 (right graph).
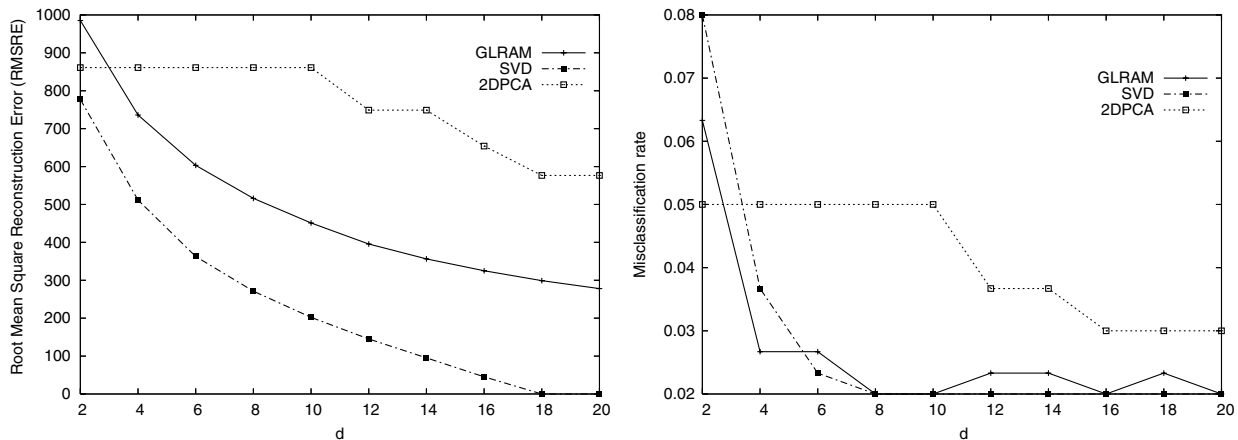

Figure 2. Comparison of reconstruction error (left) and misclassification rate (right) on PIX.
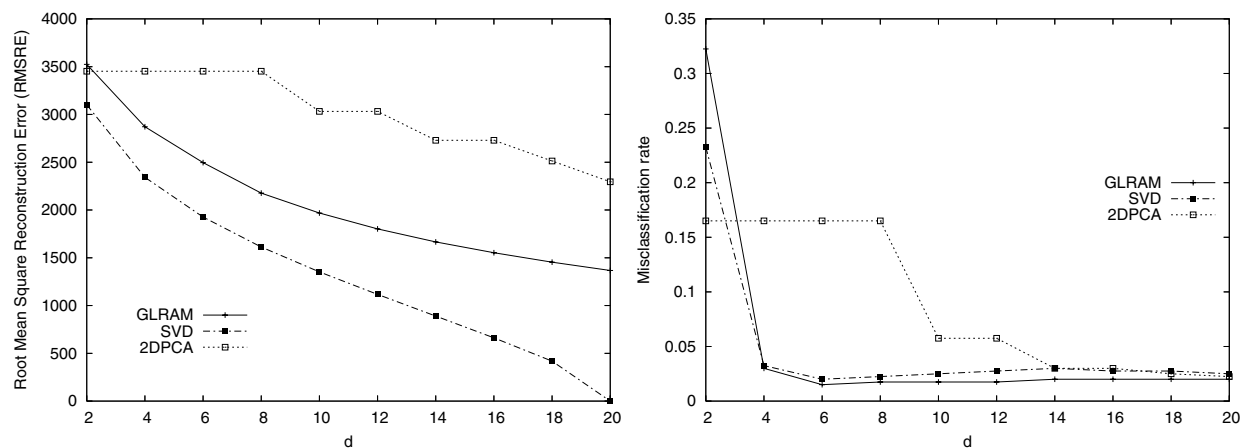

Figure 3. Comparison of reconstruction error (left) and misclassification rate (right) on ORL. 

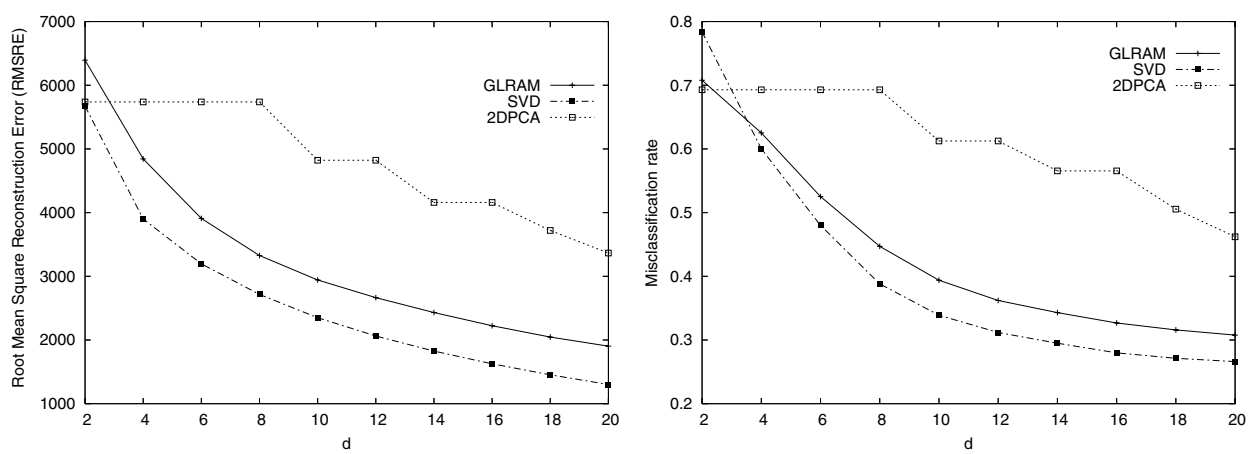

Figure 4. Comparison of reconstruction error (left) and misclassification rate (right) on AR.
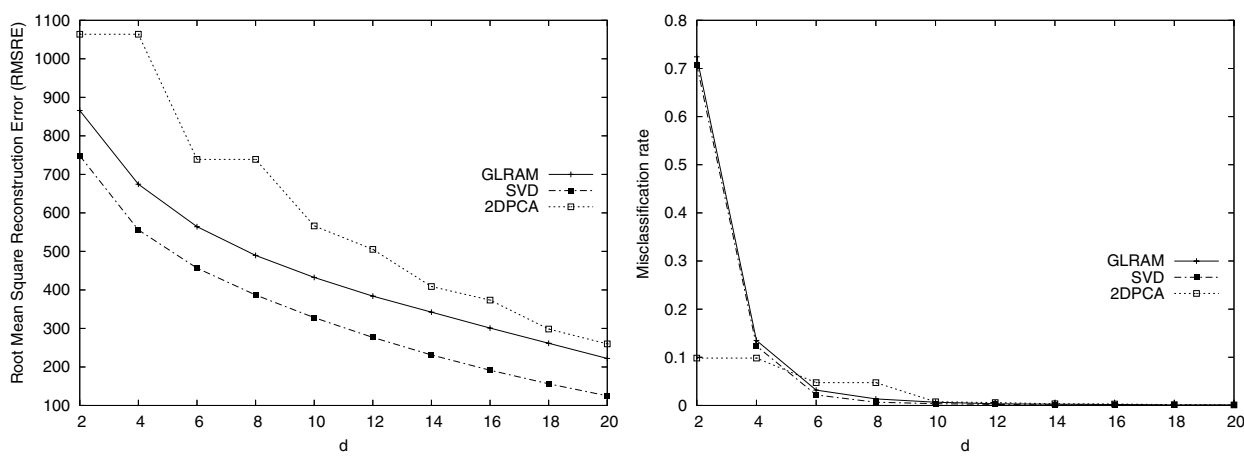

Figure 5. Comparison of reconstruction error (left) and misclassification rate (right) on PIE.

- GLRAM is competitive with SVD for classification in most cases, even though GLRAM has larger RMSRE values. This may be related to the fact that GLRAM is able to utilize the locality information (e.g. smoothness in an image) intrinsic in the image, which leads to good classification performance. We apply GLRAM to datasets without any locality property, such as text documents and gene expression data, by reshaping each vector as a matrix. GLRAM performs quite poorly in both the reconstruction error and classification as compared to SVD.

- The reconstruction error and misclassification rate on AR are much higher than those of other image datasets. This may be related to the large within-class variances on AR, due to the presence of sun glasses and scarves, as mentioned in Section 5.1.

\subsection{Compression effectiveness}

In this experiment, we examine the quality of the images compressed by the proposed algorithm and compare it with SVD and 2DPCA. Image compression is commonly applied as a pre-processing step for storage and transmission of large image data. There exists a tradeoff 

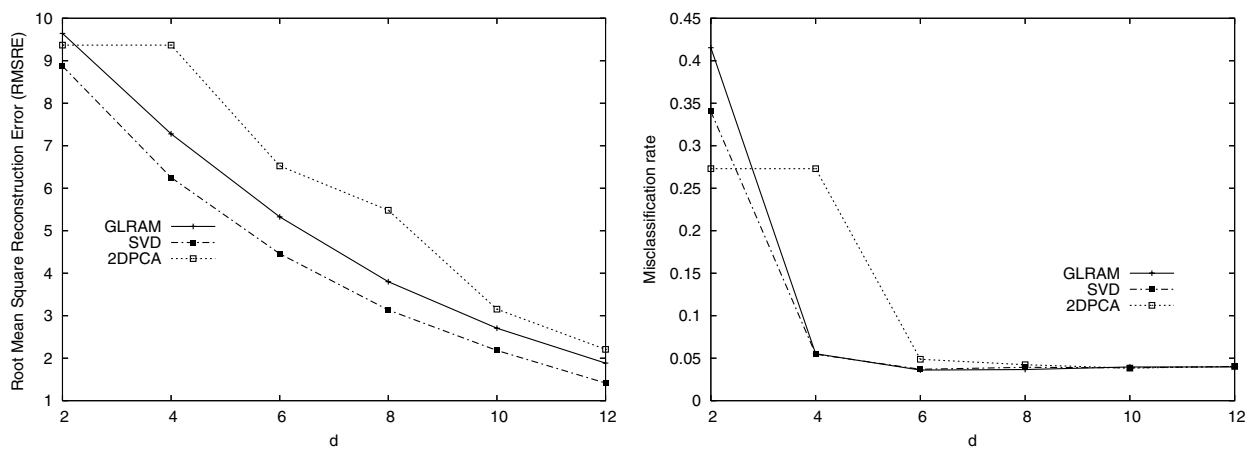

Figure 6. Comparison of reconstruction error (left) and misclassification rate (right) on USPS.
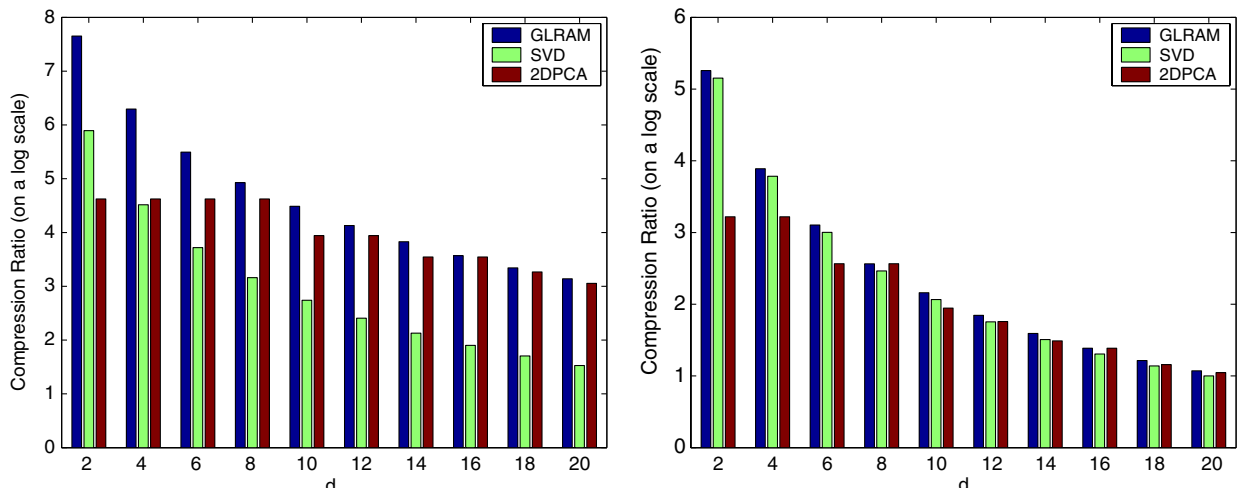

Figure 7. Comparison of compression ratio (on a log scale) on AR (left) and PIE (right). The horizontal axis denotes the value of $d$, and the vertical axis denotes the compression ratio (on a log scale).

between quality of compressed images and compression ratio, as a high compression ratio usually leads to poor quality of compressed images.

Figure 8 shows images of 10 different persons from the ORL dataset. The 10 images in the first row are the original images from the dataset. The 10 images in the second row are the ones compressed by the GLRAM algorithm with $d=10$. The compression ratio is about 98.0. The images compressed by SVD and 2DPCA with approximately the same number of reduced dimensions as GLRAM are shown in the third and fourth rows of figure 8 respectively. It is clear that the images compressed by our proposed algorithm have slightly better visual quality than those compressed by 2DPCA, while the ones compressed by SVD have the best visual quality. However, the compression ratio of SVD (3.85) is much smaller than that of GLRAM (98.0).

Figure 9 shows images of 10 different digits from the USPS dataset. $d=5$ is used in GLRAM. The compression ratio is about 10 . GLRAM and SVD perform slightly better than 2DPCA. Furthermore, the compression ratio of SVD (9.4) is close to that of GLRAM 
(10.2). The different behavior between ORL and USPS is related to the fact that USPS has a relatively large number of data points compared to its dimension, i.e., $n \gg r c$.

\section{6. $G L R A M+S V D$}

In this experiment, we study the combination of GLRAM and SVD, namely GLRAM + SVD, where the dimension is further reduced by SVD. More specifically, in the first stage, each data point $A_{i} \in \mathbb{R}^{r \times c}$ is reduced to $M_{i} \in \mathbb{R}^{d \times d}$ by GLRAM, with

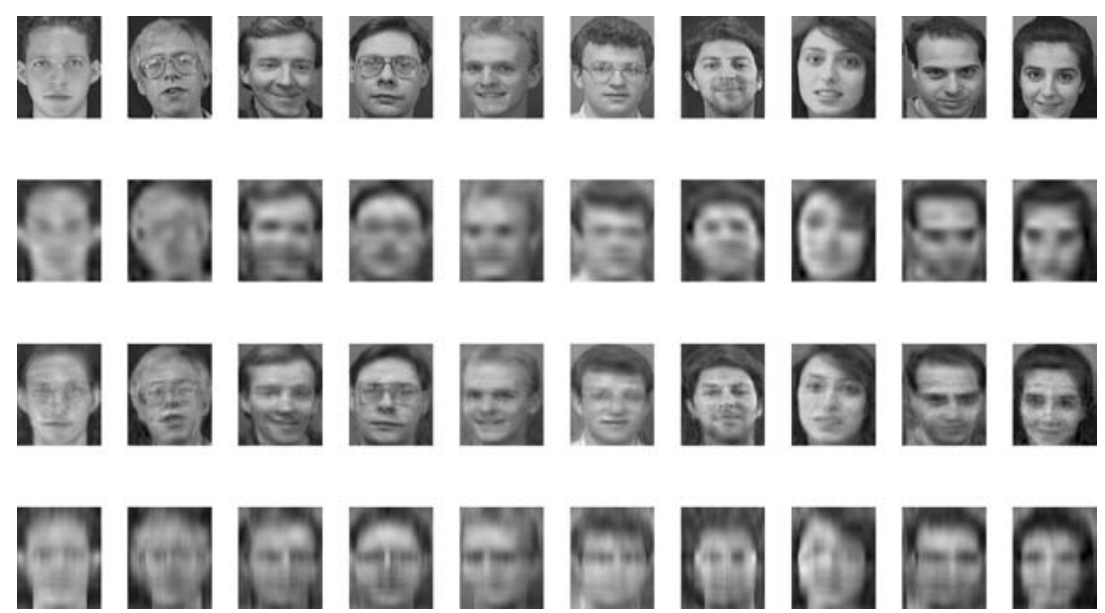

Figure 8. First row: raw images from ORL dataset. Second row: images compressed by GLRAM. Third row: images compressed by SVD. Fourth row: images compressed by 2DPCA. Note that the compression ratio of SVD (3.85) is much smaller than that of GLRAM (98.0).

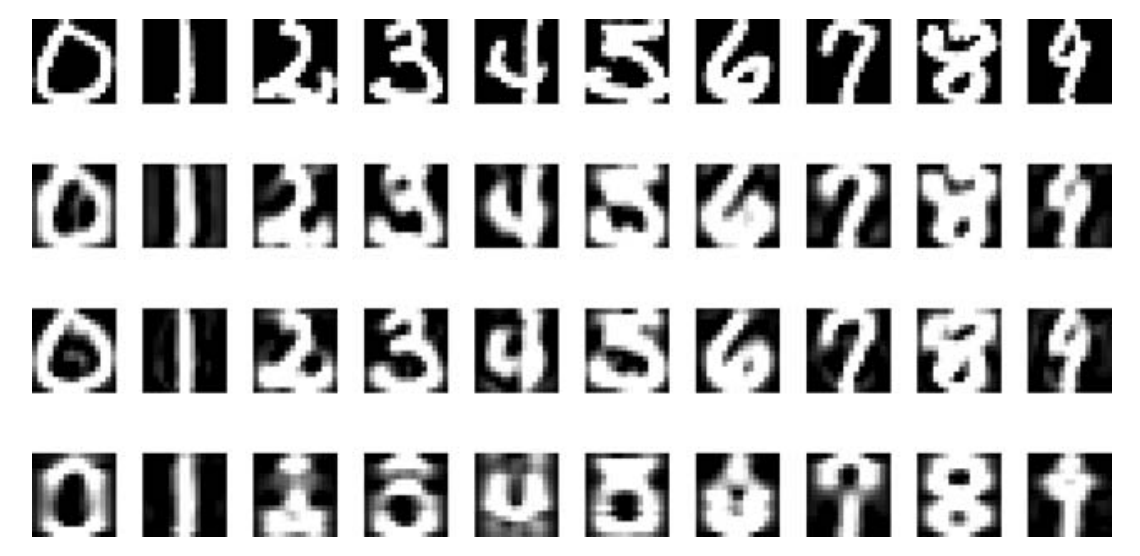

Figure 9. First row: raw images from USPS dataset. Second row: images compressed by GLRAM. Third row: images compressed by SVD. Fourth row: images compressed by 2DPCA. 
$d<\min (r, c)$. In the second stage, each $M_{i}$ is first transformed to a vector $v_{i} \in \mathbb{R}^{d^{2}}$ by matrix-to-vector alignment, where a matrix is transformed to a vector by concatenating all its rows together consecutively. Then $v_{i}$ is further reduced to $v_{i}{ }^{L} \in \mathbb{R}^{k}$ by SVD with $k<d^{2}$. The flowchart of the GLRAM + SVD algorithm is shown in figure 10 graphically.

The complexity of the first (GLRAM) stage is $O\left(I(r+c)^{2} d n\right)$, where the number of iterations $I$ is usually small. The second stage applies SVD to a $n$ by $d^{2}$ matrix, hence takes $O\left(n d^{2} \min \left(n, d^{2}\right)\right)$. Therefore, the total time complexity of GLRAM + SVD is $O(n d$ $\left.\left((r+c)^{2}+\min \left(n d, d^{3}\right)\right)\right)$. Assuming $r \approx c \approx \sqrt{N}$, the time complexity is simplified to

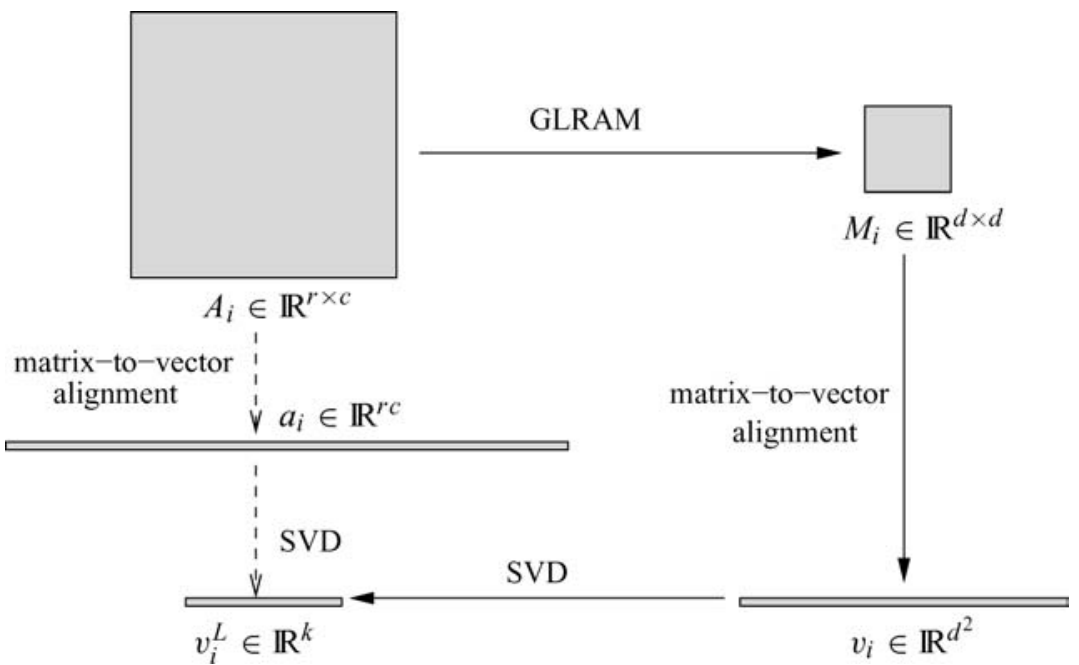

Figure 10. Flowchart of the GLRAM + SVD algorithm (solid lines). It has two stages: In the first stage, each data point $A_{i} \in \mathbb{R}^{r \times c}$ is transformed to $M_{i} \in \mathbb{R}^{d \times d}$; In the second stage, $M_{i}$ is first transformed to a vector $v_{i} \in$ $\mathbb{R}^{d^{2}}$ by matrix-to-vector alignment, which is further reduced to a vector $v_{i}{ }^{L} \in \mathbb{R}^{k}$ by SVD. Note that $d<\min (r$, $c$ ) and $k<d^{2}$. For traditional SVD (dashed lines), $A_{i} \in \mathbb{R}^{r \times c}$ is first transformed to a vector $a_{i} \in \mathbb{R}^{r c}$, which is then reduced to $v_{i}{ }^{L} \in \mathbb{R}^{k}$ by SVD directly.
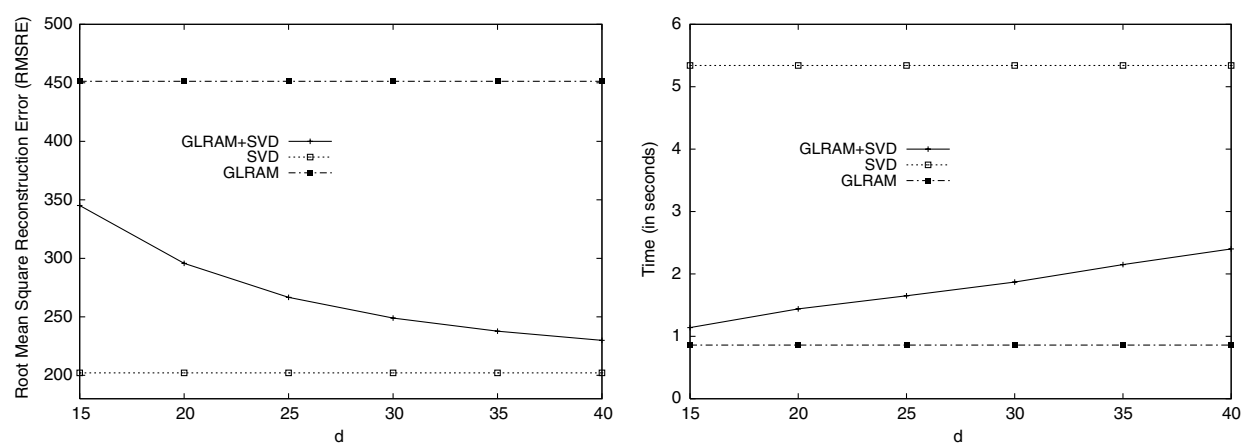

Figure 11. Comparison of reconstruction error (left) and computation time (right) on PIX. 

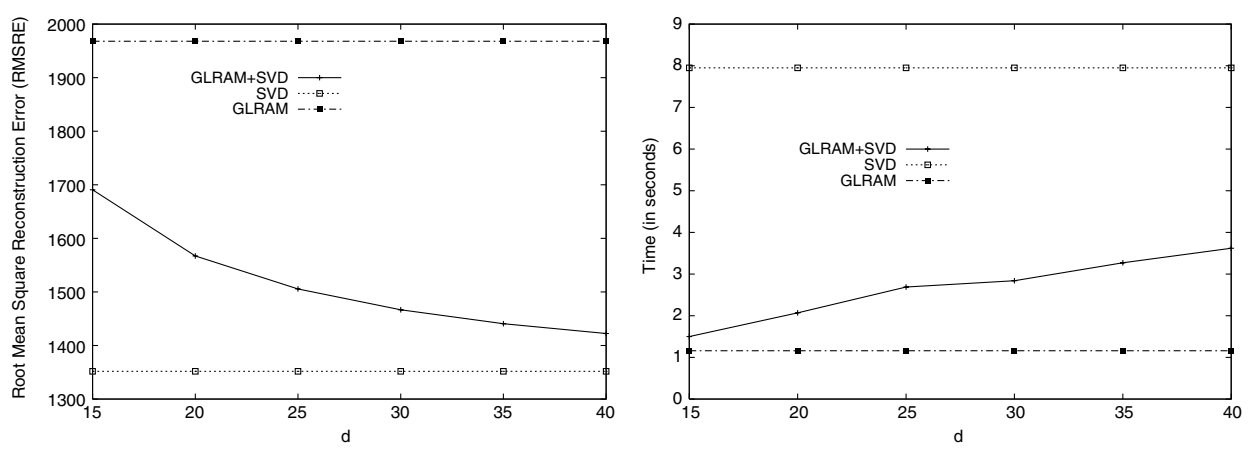

Figure 12. Comparison of reconstruction error (left) and computation time (right) on ORL.
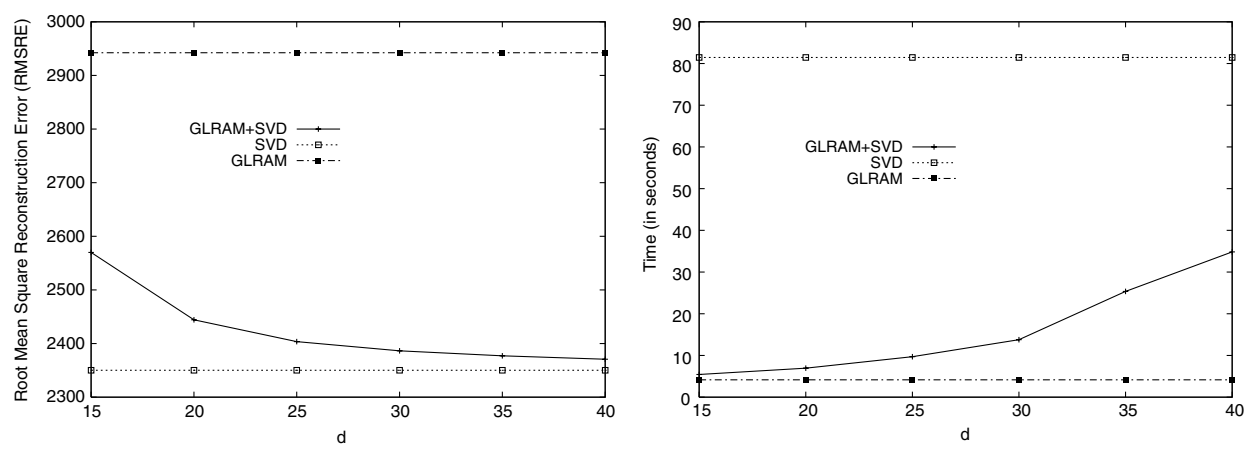

Figure 13. Comparison of reconstruction error (left) and computation time (right) on AR.

$O\left(n d\left(N+\min \left(n d, d^{3}\right)\right)\right)$. Note that both the GLRAM and SVD stages in GLRAM + SVD have much smaller computation costs than SVD, especially when $d$ is small. (Note that the cost of SVD on an $n \times N$ matrix is $O(n N \min (n, N))$.)

We apply GLRAM + SVD to the image datasets and compare it with SVD and GLRAM in terms of reconstruction error and computation time, when using the same number of reduced dimensions. For simplicity, we use $k=100$ in SVD for PIX, ORL and AR and $k=$ 25 for PIE and USPS. Hence, the reduced dimension of GLRAM and GLRAM + SVD is 100 (or 25). The value of $d$ determines the intermediate dimension of the GLRAM stage in GLRAM + SVD. We examine the effect of $d$ on the performance of GLRAM + SVD, and the results are summarized in figures 11-15, where the horizontal axis denotes the value of $d$ (between 15 and 40 for PIX, ORL and AR, between 6 and 16 for PIE, and between 6 and 12 for USPS) and the vertical axis denotes the reconstruction error, measured by the RMSRE value (left graph) and the computation time, measured in seconds (right graph). It is worthwhile to note that the reduced dimension of GLRAM is fixed in the comparison, while the reduced dimension of the intermediate (GLRAM) stage in GLRAM + SVD varies.

The main observations include: 

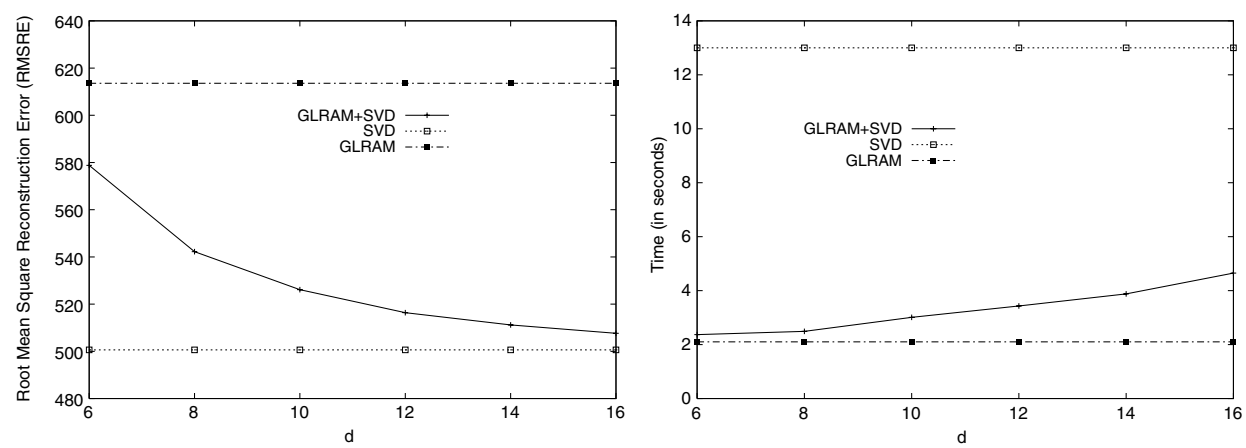

Figure 14. Comparison of reconstruction error (left) and computation time (right) on PIE.
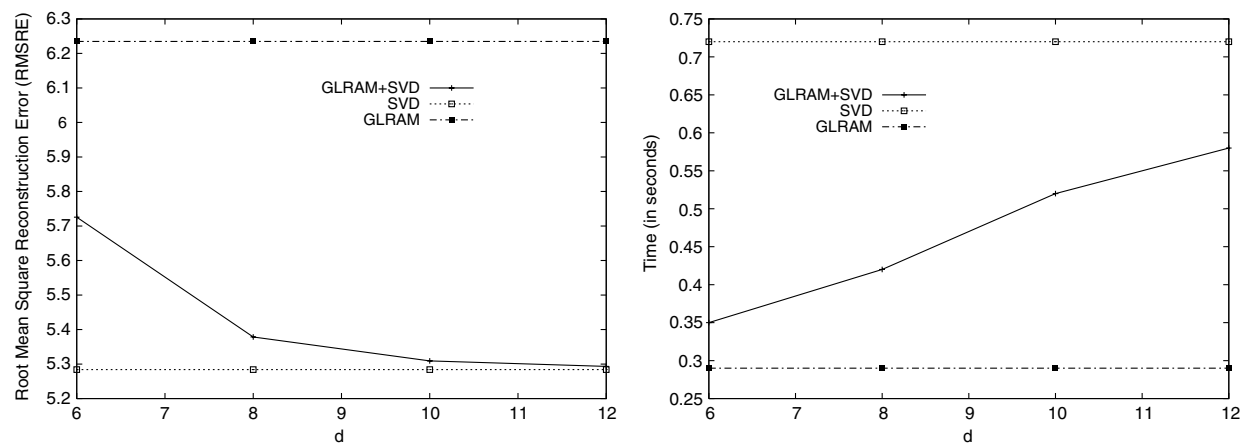

Figure 15. Comparison of reconstruction error (left) and computation time (right) on USPS.

- As $d$ increases, the RMSRE value of GLRAM + SVD decreases. By combining GLRAM and SVD, GLRAM + SVD achieves a dramatic reduction of the RMSRE value as compared to GLRAM.

- The computation time of GLRAM + SVD increases, as $d$ increases. There is a tradeoff between the reconstruction error and the computation time, when choosing the best $d$.

The above experiment shows that it may be beneficial to combine GLRAM with SVD, since it has much lower reconstruction error than GLRAM, while keeping the computation cost low. The performance of GLRAM + SVD critically depends on the value of $d$. To choose the optimal $d$, one needs to consider the tradeoff between the computation cost and the reconstruction error, as a larger value of $d$ usually leads to higher computation cost and smaller reconstruction error.

\section{Conclusions and future work}

A novel algorithm, named GLRAM, for low rank approximations of a collection of matrices is presented. The algorithm works in an iterative and interleaved fashion and the approximation is improved during successive iterations. Experimental results show that 
the algorithm converges rapidly. Detailed analysis shows that GLRAM has asymptotically minimum space requirement and lower time complexity than SVD, which is desirable for large and high-dimensional datasets. Specifically, GLRAM involves eigenvalue problems of size $r^{2}$ or $c^{2}$, as compared to size $r c n(=N n)$ in SVD.

A natural application for GLRAM is in image compression and retrieval, where each image is represented in its native matrix form. We evaluate the proposed algorithm in terms of the reconstruction error and classification, and compare it with 2DPCA and SVD. Results show that when using the same number of reduced dimensions, the proposed algorithm is competitive with 2DPCA and SVD for classification, while GLRAM results in a larger reconstruction error than SVD. In terms of compression ratio, GLRAM outperforms SVD, especially when the number of dimensions is relatively large compared to the number of data points. However, our experiments show that GLRAM can fail both in reconstruction error and classification when the data do not have locality property (e.g. smoothness in an image), such as text documents and gene expression data. Further study is needed to show how a native data vector can be rearranged into a matrix so that related variables are spatially close.

To further reduce the reconstruction error of GLRAM, we study the GLRAM + SVD algorithm, where SVD is preceded by GLRAM. In this composite algorithm, GLRAM can be considered as a pre-processing step for SVD. Extensive experiments show that, when using the same number of reduced dimensions, GLRAM + SVD achieves a significant reduction of the reconstruction error as compared to GLRAM, while keeping the computation cost small. The reconstruction error of GLRAM + SVD is close to that of SVD, especially when the intermediate reduced dimension in the GLRAM stage is large, while it has a smaller computation cost than SVD. One of our future research directions is to understand why GLRAM has larger reconstruction error than SVD, when using the same number of reduced dimensions.

There are several other crucial questions that still remain to be answered:

- In Section 5.2, we study the effect of the ratio of $\ell_{1}$ to $\ell_{2}$ on reconstruction error. Experimental results show that choosing $\ell_{1} / \ell_{2} \approx 1$ works well in practice, even when the original row $(r)$ and column $(c)$ dimensions are quite different. It may be related to the effect of balancing between the left and right transformations involved in GLRAM. However, a rigorous theoretical justification behind this is still not available.

- In Section 5.3, we study the convergence property of GLRAM and the sensitivity of GLRAM to the choice of the initial $L_{0}$. Extensive experiments show that for image datasets, GLRAM may converge to the global solution, regardless of the choice of the initial $L_{0}$. However, it is not true in general, as shown in the RAND dataset. The remaining question is whether there exist certain conditions on the $A_{i}$ 's, under which GLRAM has the global convergence property.

\section{Acknowledgment}

We thank the Associate Editor and the reviewers for helpful comments that greatly improved the paper. We also thank Prof. Ravi Janardan and Dr. Chris Ding for helpful discussions. 
This research is sponsored, in part, by the Army High Performance Computing Research Center under the auspices of the Department of the Army, Army Research Laboratory cooperative agreement number DAAD19-01-2-0014, the content of which does not necessarily reflect the position or the policy of the government, and no official endorsement should be inferred. Support Fellowships from Guidant Corporation and from the Department of Computer Science \& Engineering, at the University of Minnesota, Twin Cities is gratefully acknowledged.

\section{Notes}

1. Here the compression ratio means the percentage of space saved by the low rank approximations to store the data. Details can be found in Sections 2 and 3.

2. http://peipa.essex.ac.uk/ipa/pix/faces/manchester/test-hard/

3. http://www.uk.research.att.com/facedatabase.html

4. http://rvl1.ecn.purdue.edu/ aleix/aleix_face_DB.html

5. http://www.ri.cmu.edu/projects/project_418.html

6. http://www-stat-class.stanford.edu/ tibs/ElemStatLearn/data.html

\section{References}

Achlioptas, D., \& McSherry, F. (2001). Fast computation of low rank matrix approximations. In ACM STOC Conference Proceedings (pp. 611-618).

Aggarwal, C. C. (2001). On the effects of dimensionality reduction on high dimensional similarity search. In $A C M$ PODS Conference Proceedings (pp. 256-266).

Averbuch, A., Lazar, D., \& Israeli, M. (1996). Image compression using wavelet transform and multiresolution decomposition. IEEE Transactions on Image Processing, 5:1, 4-15.

Berry, M., Dumais, S., \& O’Brie, G. (1995). Using linear algebra for intelligent information retrieval. SIAM Review, 37, 573-595.

Bjork, A., \& Golub, G. (1973). Numerical methods for computing angles between linear subspaces. Mathematics of Computation, 27:123, 579-594.

Brand, M. (2002). Incremental singular value decomposition of uncertain data with missing values. In $E C C V$ Conference Proceedings (pp. 707-720).

Castelli, V., Thomasian, A., \& Li, C.-S. (2003). CSVD: Clustering and singular value decomposition for approximate similarity searches in high dimensional space. IEEE Transactions on Knowledge and Data Engineering, $15: 3,671-685$.

Deerwester, S., Dumais, S., Furnas, G., Landauer, T., \& Harshman, R. (1990). Indexing by latent semantic analysis. Journal of the Society for Information Science, 41, 391-407.

Dhillon, I., \& Modha, D. (2001). Concept decompositions for large sparse text data using clustering. Machine Learning, 42, 143-175.

Drineas, P., Frieze, A., Kannan, R., Vempala, S., \& Vinay, V. (1999). Clustering in large graphs and matrices. In ACM SODA Conference Proceedings (pp. 291-299).

Duda, R., Hart, P., \& Stork, D. (2000). Pattern classification. Wiley.

Edelman, A., Arias, T. A., \& Smith, S. T. (1998). The geometry of algorithms with orthogonality constraints. SIAM Journal on Matrix Analysis and Applications, 20:2, 303-353.

Frieze, A., Kannan, R., \& Vempala, S. (1998). Fast monte-carlo algorithms for finding low-rank approximations. In ACM FOCS Conference Proceedings (pp. 370-378).

Fukunaga, K. (1990). Introduction to statistical pattern classification. San Diego, California, USA: Academic Press.

Golub, G. H., \& Van Loan, C. F. (1996). Matrix computations, 3rd edition. Baltimore, MD, USA: The Johns Hopkins University Press. 
Gu, M., \& Eisenstat, S. C. (1993). A fast and stable algorithm for updating the singular value decomposition. Technical Report YALEU/DCS/RR-966, Department of Computer Science, Yale University.

Kanth, K. V. R., Agrawal, D., Abbadi, A. E., \& Singh, A. (1998). Dimensionality reduction for similarity searching in dynamic databases. In ACM SIGMOD Conference Proceedings (pp. 166-176).

Kleinberg, J., \& Tomkins, A. (1999). Applications of linear algebra in information retrieval and hypertext analysis. In ACM PODS Conference Proceedings (pp. 185-193).

Kolda, T. G. (2001). Orthogonal tensor decompositions. SIAM Journal on Matrix Analysis and Applications, 23:1, 243-255.

Martinez, A., \& Benavente, R. (1998). The AR face database. Technical Report CVC Tech. Report No. 24.

Papadimitriou, C. H., Tamaki, H., Raghavan, P., \& Vempala, S. (1998). Latent semantic indexing: A probabilistic analysis. In PODS Conference Proceedings (pp. 159-168).

Samaria, F., \& Harter, A. (1994). Parameterisation of a stochastic model for human face identification. In Proceedings of 2nd IEEE Workshop on Applications of Computer Vision, Sarasota FL (pp. 138-142).

Shashua, A., \& Levin, A. (2001). Linear image coding for regression and classification using the tensor-rank principle. In CVPR Conference Proceedings (pp. 42-49).

Sim, T., Baker, S., \& Bsat, M. (2004). The CMU pose, illumination, and expression (PIE) database. IEEE Transactions on Pattern Analysis and Machine Intelligence, 25:12, 1615-1618.

Srebro, N., \& Jaakkola, T. (2003). Weighted low-rank approximations. In ICML Conference Proceedings (pp. 720-727).

Vasilescu, M. A. O., \& Terzopoulos, D. (2002). Multilinear analysis of image ensembles: Tensorfaces. In ECCV Conference Proceedings (pp. 447-460).

Yang, J., Zhang, D., Frangi, A., \& Yang, J. (2004). Two-dimensional PCA: A new approach to appearance-based face representation and recognition. IEEE Transactions on Pattern Analysis and Machine Intelligence, 5:1, 131-137.

Zhang, T., \& Golub, G. H. (2001). Rank-one approximation to high order tensors. SIAM Journal on Matrix Analysis and Applications, 5:2, 534-550.

Received May 25, 2004

Revised June 28, 2005

Accepted June 28, 2005 